\section{HONOURS, AWARDS, APPOINTMENTS}

Practice of the month

Evesham Dental Health Team is the Mouth Cancer Foundation's Practice of the Month for December. The team has proven they go above and beyond to implement the charity's Mouth Cancer Screening Accreditation Scheme.

\section{New president}

Implant and cosmetic dentist Zaki Kanaan has been elected President of the British Academy of Cosmetic Dentistry (BACD) for the term 2013-2014.

\section{Implant president}

Dr Philip Friel has taken over the presidency of the Association of Dental Implantology (ADI) for the 2013-2015 term. Dr Friel has an implant referral clinic in Glasgow and has had an active involvement with the ADI for seven years.

\section{Member from Wales}

Kirstie Moons has been appointed the GDC member from Wales, which completes the new 12-member body which will lead the GDC. Kirstie started her career as a dental nurse and is currently Associate Director for DCP Education within the Dental Postgraduate section at Cardiff University, Wales Deanery and an External Examiner for the Certificate of Higher Education in Dental Nursing and the Foundation Award in Science and Dental Therapy at Portsmouth University.

\title{
HELP SLOW THE GLOBAL GROWTH OF ANTIBIOTIC RESISTANCE
}

Dental practitioners should only consider the use of antibiotics when the drainage of an acute dental infection cannot be achieved, says the Faculty of General Dental Practice (UK). The FGDP(UK) is keen to highlight the ineffectiveness of antimicrobials on oral abscesses and the need for practitioners to ensure that they act responsibly to help slow the global development of antibiotic resistance.

The FGDP(UK) collaborated with the Association of Clinical Oral Microbiologists (ACOM) and the British Society for Antimicrobial Chemotherapy on a 'Thunderclap initiative', launched to coincide with European Antibiotic Awareness Day on 18 November 2013. The Thunderclap, which harnesses individuals' social media networks to spread messages, asked supporters to pledge that "where a dental infection needs drainage, this is provided before considering the use of antibiotics. The pledge has reached over 30,000 people.

Dr Nikolaus Palmer, FGDP(UK) Board member and Editor of the
Faculty's publication Antimicrobial prescribing for general dental practitioners, ${ }^{1}$ said: 'The FGDP(UK)'s evidence-based guidance sets out sensible protocols for dental practitioners when considering the need to prescribe antimicrobials. Those that follow this guidance will already be aware that the majority of uncomplicated dental swellings can be successfully treated by removal of the source of the infection by drainage of the associated abscess. It is vital that dental practitioners recognise their role in helping to reduce the development of antimicrobial resistance.'

Antimicrobial resistance has become a worldwide problem over the last few decades and now constitutes a major threat to public health. Dentists working in the NHS in England prescribe nearly $10 \%$ of all oral antimicrobials in the primary care setting, and all antibiotics prescribed in dentistry can have an adverse effect on the rest of the body's microbial flora.

1. Palmer N (ed). Antimicrobial prescribing for general dental practitioners, 2nd ed. Faculty of General Dental Practitioners (UK), 2012.

\section{GUM DISEASE INCREASES ASTHMA RISK}

New research has identified that, despite age, body mass index and smoking habits, people with gum disease are still at risk of developing asthma, ${ }^{1}$ a condition that claims three lives every day in the UK.

The study looked at 220 people; 113 had asthma, 107 did not. After being diagnosed with gum
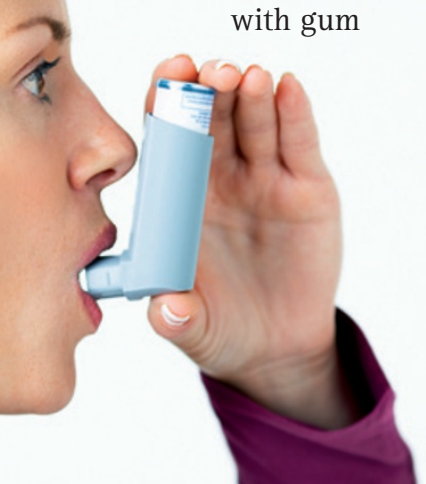

disease, researchers accounted for age, schooling level, osteoporosis, smoking habit and body mass index and still found that adults with gum disease were approximately five times more likely to develop asthma than those without gum disease.

According to Asthma UK, ${ }^{2}$ 5.4 million people in the UK are currently receiving treatment for asthma. The UK has some of the highest rates of asthma across Europe, a fact that could be linked to UK attitudes towards oral health.

The British Dental Health Foundation estimates that less than half of all adults have an acceptable oral hygiene routine. Figures from the Adult Dental
Health Survey ${ }^{3}$ revealed that basic dental products aren't being used as part of an all-round routine. Only three in ten (31\%) people use mouthwash and less than one in four (22\%) use floss.

The same data also showed $42 \%$ of adults only use a toothbrush and toothpaste, with more than one in four (27\%) saying they use an electric brush. Furthermore, it also showed how less than one in four adults (24\%) do not know what level of fluoride their toothpaste should contain.

Chief Executive of the British Dental Health Foundation, Dr Nigel Carter OBE, said: 'The research points to a significant association between gum disease and asthma. Asthma is in a long line of health problems linked to gum disease that includes heart problems, dementia, pregnancy complications and pancreatic cancer'.

1. Gomes-Filho I S, Soledade-Marques K R, Seixas da Cruz S et al. Does periodontal infection have an effect on severe asthma in adults? J Periodonto/ 2013; DOI: 10.1902/jop.2013.130509.

2. Asthma UK. Asthma facts and FAOs. www.asthma.org.uk/ asthma-facts-and-statistics.

3. Adult Dental Health Survey 2009 - Summary report and thematic series. 24 March 2011. 